\title{
Are DMSA Scans Reliable for Diagnosing Vesicoureteral Reflux in Infants and Children?
}

\author{
Saeed Bafaraj, Remaz Alahmadi \\ Department of Diagnostic Radiology, King Abdulaziz University, Jeddah, KSA \\ Email: smbafaraj@kau.edu.sa \\ Received 27 September 2014; revised 24 October 2014; accepted 24 November 2014 \\ Copyright (C) 2014 by authors and Scientific Research Publishing Inc. \\ This work is licensed under the Creative Commons Attribution International License (CC BY). \\ http://creativecommons.org/licenses/by/4.0/ \\ (c) (i) Open Access
}

\section{Abstract}

A retrospective study for data record of 106 patients subjected to voiding cystourethrogram test (VCUG), sonography test, and dimercaptosuccinic acid (DMSA) scintigraphy take place at King Abdulaziz University, radiology department to find out the possibilities of detecting vesicoureteral reflux (VUR) in infants and children. Hydronephrosis \& Renal pelvis dilatation were the highest frequency reason for VUR scan study and represent $36 \%$ of the overall cases with $53 \%$ positive finding, while Recurrent UTI frequency was $22.6 \%$ with $83 \%$ positive finding, and patients who had history of VUR come at the third place with $15 \%$ frequency and $81 \%$ positive finding. VUR was found in 65 of 106 children representing $61 \%$. Male children are more likely to diagnostic with VUR than female and they represented $69 \%$ while $31 \%$ for female. Among the children with VUR, 39 had high-grade reflux (grade IV or V), while 26 had low-grade reflux (grades I, II or III). When we classified VUR into low-grade and high-grade, we found the majority of children with highgrade VUR had an abnormal DMSA and represented 77.4\%, while 13 children with low-grade VUR had normal study. These studies are agreed with the present study; most children with normal DMSA scan finding had low grade reflux. In this study, 48 of 65 children (74\%) the DMSA scintigraphy shows abnormal findings during the period of UTI while the VCUG scan study shows VUR in $\mathbf{7 5 \%}$ of the children during the period of UTI. Comparing results of ultrasound and VCUG scan, $59 \%$ of the ultrasound findings gave the same result as VCUG in detecting the VUR in children. Then we confirm it all with the urine culture results of the same children. We found the majority of children with VUR had abnormal US finding during the period of UTI and represented $83 \%$.

\section{Keywords}

Voiding Cystourethrography (VCUG), DMSA, Vesicoureteral Reflux 


\section{Introduction}

Vesicoureteral reflux (VUR) is common in infants and children. It's represents the retrograde flow of urine from the bladder to the upper urinary tract. Voiding cystourethrogram (VCUG) should be done in children to diagnose VUR, but there seems to be controversy regarding the role of VCUG in the assessment of these children. Scintigraphy of Renal and ultrasound can help to diagnose the VUR.

\section{Objectives}

To find out the possibilities of Dimercaptosuccinic acid (DMSA) scintigraphy and ultrasound in detecting vesicoureteral reflux (VUR) in infants and children and if that can replace the voiding cystourethrography (VCUG).

\section{Literature Review}

\subsection{Anatomy}

The urinary system consists of the Kidneys, the ureters, the urinary bladder, and the urethra. The position of the left kidney extends superiorly slightly more than the right kidney. Both lie retroperitoneal in the superior lumbar region. The kidney produce urine and during urination urine is forced out of the body. A kidney has superficial cortex, a deeper medulla consisting mainly of medullary pyramid, and medial pelvis. Extension of pelvis (calyces) surrounds and collects urine draining from the apices of the medullary pyramids. The hilus is enter for the renal artery and exit for the renal vein and ureter. The ureter communication with the renal pelvis and extend to the urinary bladder. Peristaltic contractions by smooth muscles move the urine. The bladder includes the trigon, the neck and the internal urethral sphincter in both gender, the urethra passes through urogenital diaphragm a circular band of skeletal muscles forms the external urethral sphincter, that under voluntary control. The nerve supply of the kidneys is derived from the renal plexus [1].

\subsection{Physiology}

The function of urinary system are eliminating organic waste products, regulating plasma concentration of ions, regulating blood volume and pressure by adjusting the volume of water lost and releasing hormones, helping to stabilize blood $\mathrm{pH}$ and conserving nutrients. The process of urination is called micturition reflux, its emptying of the bladder which is initiated by stretch receptors in the bladder wall. Voluntary urination involves coupling this reflex with voluntary relaxation of the external sphincter, allows the opening of the internal sphincter [2].

\subsection{Anatomical Abnormality}

\subsubsection{Posterior Urethral Valves}

Congential valves in the posterior urethra in boys are commonest causes of bladder outflow obstruction in male children. It is an obstructing membrane in the posterior male urethra as a result of abnormal in utero development. After birth, ultrasound confirms bilateral hydronephrosis and hydroureters and a thick walled bladder. They are easily demonstrated at VCUG, where dilatation of the posterior urethra is seen which terminates abruptly in convex border formed by the valves [3]

\subsubsection{Neurogenic Bladder}

There are two basic types of neurogenic bladder. First, the large atonic smooth walled bladder with poor or absent contractions and a large residual volume. Second, the hypertrophic type which can be regarded as neurologically induced bladder outflow obstruction. In this condition, the bladder is of small volume, has a very thick, grossly wall. The ureters and pelvicaliceal system may be dilated. Full assessment of neuropathic bladder dysfunctional requires VCUG [3].

\subsection{Pathology}

Vesicoureteral reflux (VUR) is an abnormal movement of urine from the bladder into ureters or kidneys. In most of the cases, it accrues due to the abnormality of the vesicoureteral junction or a short distal ureteric submucosal 
tunnel in the urinary bladder that change the function of the valve mechanism [4]. VUR may related with other congenital anomalies such as neurogenic bladder and posterior urethral valves in male [5]. VUR predisposes to renal infection because it carries bacteria from the bladder to the upper urinary tract [6]. Children with VUR will develop renal scars [7]. Primary VUR in children is usually diagnosed after urinary tract infection (UTI) [8] and secondary VUR occurs with posterior urethral valves or neurogenic bladder [9].

\subsection{Vesicoureteral Reflux}

Vesicoureteral Reflux (VUR) is a congenital urinary tract defect caused by the failure of the ureter to insert correctly into the bladder. It occurs in up to $1 \%$ of the general population and is associated with recurrent urinary tract infections and reflux nephropathy [10]. The primary diagnostic procedure for evaluation of VUR has been Voiding Cystourethrography (VCUG) [11]. It has many side-effects, such as pain, UTI and high radiation exposure.

\subsection{Role of VCUG}

To evaluation of vesicoureteral reflux the primary diagnostic study is VCUG. Its most be performed after the first urinary tract infection or diagnostic by hydronephrosis. This procedure also provides a study of the urethra and evaluation the patient's ability to urinate, therefore it is a functional study of bladder and urethra. VCUG should use to document the presence of VUR and determine the grade level of reflux and whether happens during voiding or during bladder filling [12]. Grades is classified into low-grade reflux (grades I, II or III) and the most severe is high-grade reflux (grade IV or V), usually coincides with the peak of voiding [12]. It shows in Table 1 and Figure 1.

Table 1. International classification of vesicoureteral reflux [13].

\begin{tabular}{cc}
\hline Grade & Characteristics \\
I & Reflux into non-dilated ureter \\
II & Reflux into the renal pelvis and calyces without dilatation \\
III & Mild/moderate dilatation of the ureter, renal pelvis and calyces with minimal blunting of the fornices. \\
IV & Dilation of the renal pelvis and calyces with moderate ureteral tortuosity. \\
V & Gross dilatation of the ureter, pelvis and calyces; ureteral tortuosity; loss of papillary impressions \\
\hline
\end{tabular}

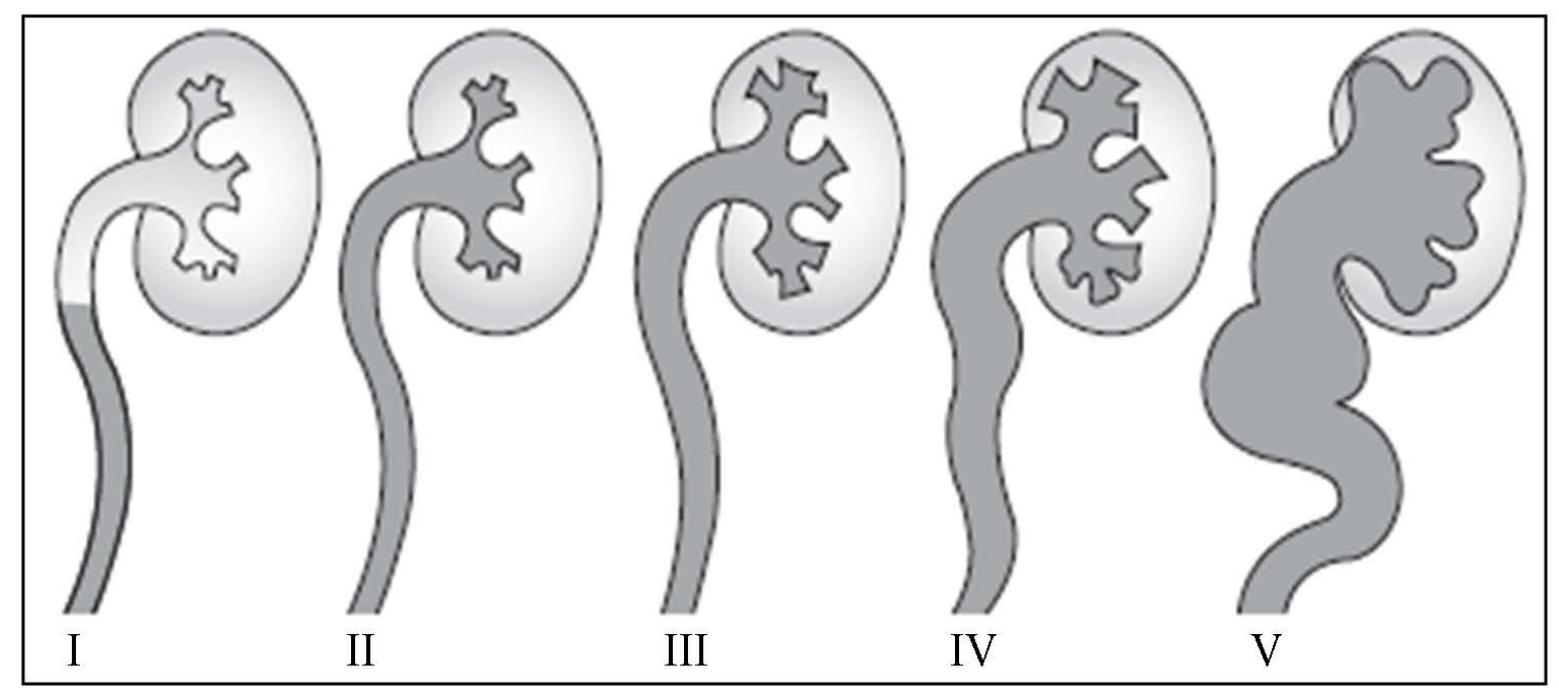

Figure 1. International classification of vesicoureteral reflux [13]. 


\subsection{Clinical Presentation (Figures 2-6)}

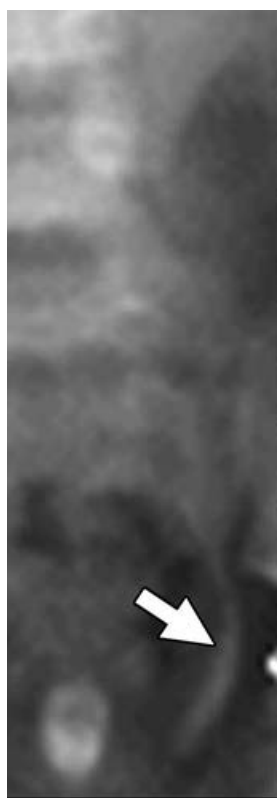

(a)

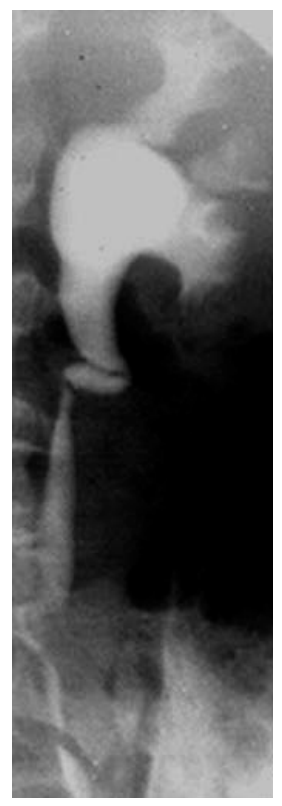

(b)

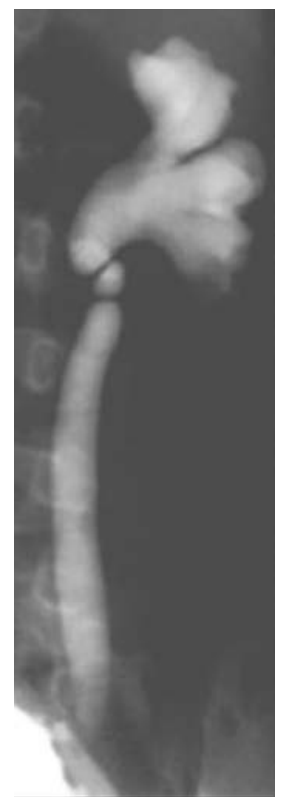

(c)

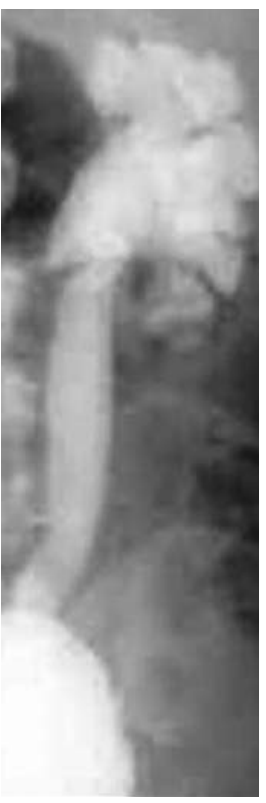

(d)

Figure 2. VCUG imaging show the different grade of the vesicoureteral reflux. (a) grade II contrast seen in the distal ureter, (b) grade III; (c) grade IV; (d) grade V [14].



Figure 3. VCUG image of a child shows back up of contrast form urinary bladder to the right ureter and collecting system [15]. 


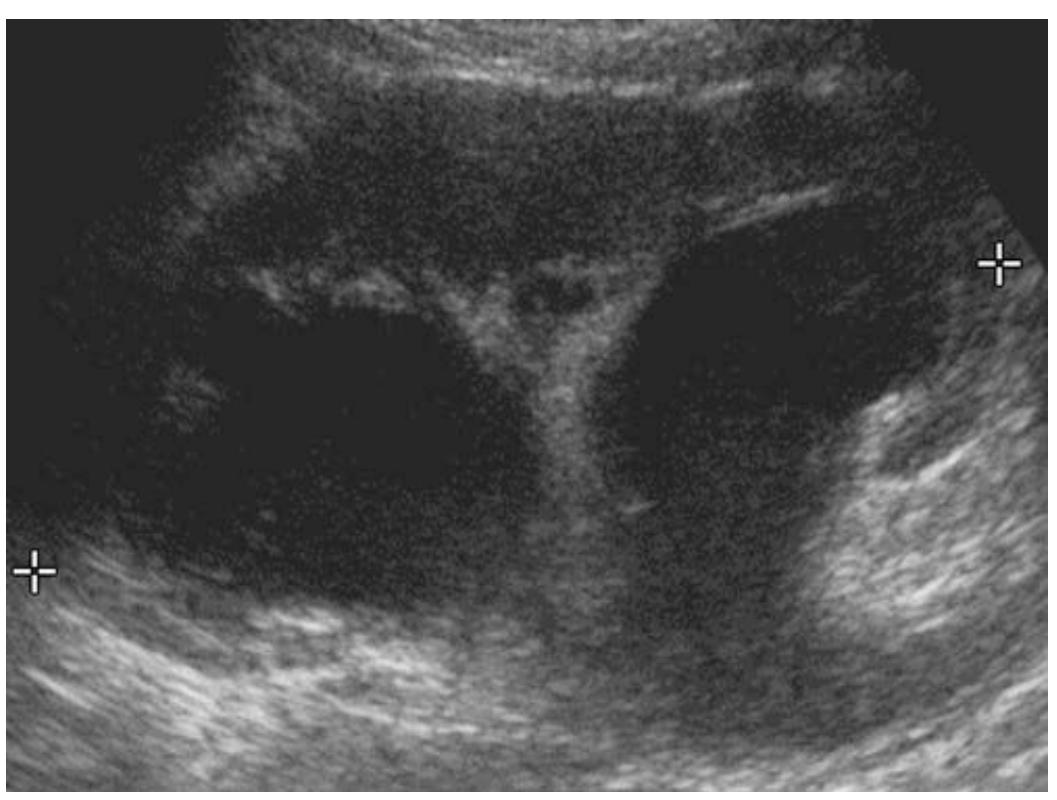

Figure 4. Ultrasound image of the kidney shows hydronephrosis [14].

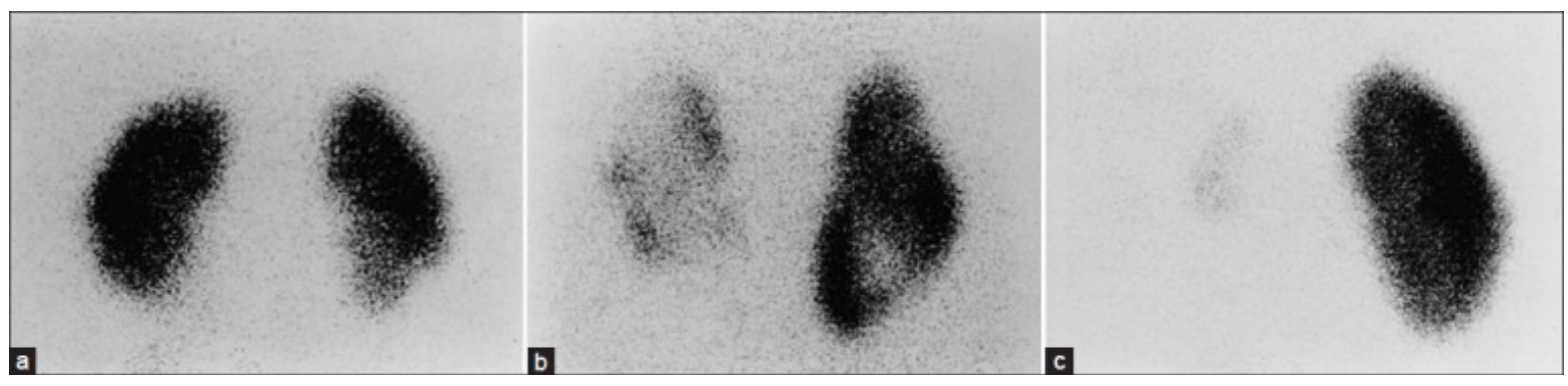

Figure 5. Different Images of the renal scarring. (a) Grade I scarring of the lower pole of the right kidney \& normal left kidney; (b) Grade II right kidney \& grade III left kidney scarring; and (c) Grade IV scarring left kidney \& normal right kidney [16].

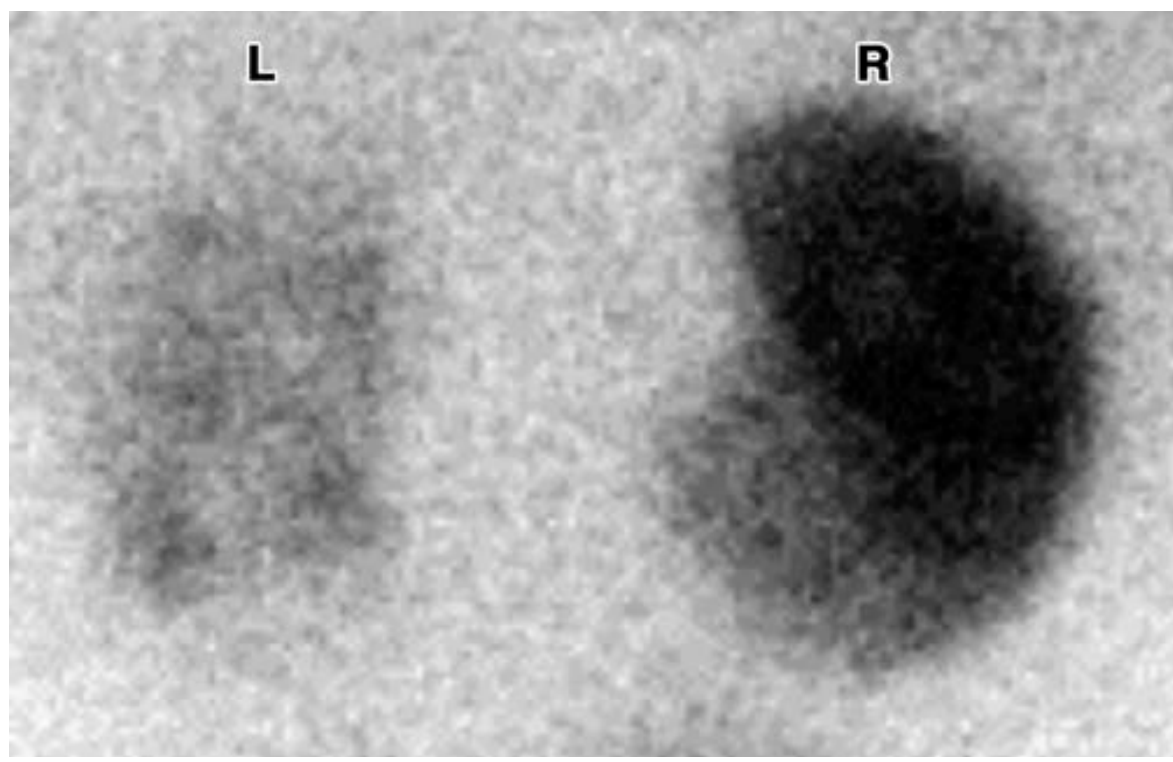

Figure 6. 99mTc-DMSA renal scintigraphy of child with history of UTI shows extensive left kidney defects [14]. 


\subsection{Radiological Examination}

\subsubsection{The Dimercaptosuccinic Acid Renal Scan}

DMSA renal scan is a noninvasive test that allows for cortical imaging; main indication is scarring from urinary tract infections because of its high sensitivity for detection of renal parenchymal injury [17]. Dimercaptosuccinic acid is a radioactive substance that is injected intravenously and the patient scanned 3 hours post injection [18]. Table 2 shows the effective dose of DMSA scan and voiding cycstourethrography.

\subsubsection{Ultrasound}

US examinations are non-invasive technique and don't involve ionizing radiation. It uses a high frequency sound required for diagnostic medical imaging in the range $1-20 \mathrm{MHz}$ and this makes it suitable for pediatric. US can be useful for evaluation of the kidneys and urinary bladder and also for assessment of the dilatation, but it but it offers poor anatomical information about the urethra and the ureters [21].

\subsubsection{Voiding Cyctourethrography Scan}

The current standard of care for evaluation of vesicoureteral reflux (VUR) is X-ray voiding cystourethrography (VCUG). The pediatric VUR guidelines panel for the American Urological Association urged the development of "less traumatic methods to determine whether reflux is present...as well as techniques that result in less radiation exposure" [22].

Voiding Cystourethrography (VCUG) with contrast medium came into widespread use around 1958, when fluoroscopes with image intensification and cine recording became generally available [23]. VUCG images are obtained while the patient urinates in a container. Filling of the patient's bladder with contrast is achieved either with the aforementioned retrograde gradual injection of contrast during a preceding RUG or with the insertion of a Foley catheter into the bladder. It's currently the most popular method for assessing vesicoureteral reflux VUR, an important component of evaluation of child with urinary tract infection UTI [24].

In VCUG right oblique images are obtained with the patient preferably supine and the penis resting extended along the thigh of the ipsilateral right flexed leg. This position provides adequate visualization of the whole length of the urethra, from the bladder neck to the external urethral meatus [25].

\section{- Technique}

cystography is usually performed after the transurethral placement of Foley catheter. a scout film of the bladder is obtained. Contrast media are introduced through the catheter into the bladder via gravity drip with the bottle approximately 1 meter above the table top [26].

\section{- Contrast Medium}

Megluminesalts of diatrizoate andlor iothalamate are most widely used contrast media for cystography. Megumine salts are less irritating to bladder mucosa. Soluations containing 15\% (weightlvolume) contrast media are generally optimal [5] Absorption of contrast media may occur from the bladder during a cystogram. Patients who have had a severe reaction to intravenous contrast media LOCM may be indicated [26].

\section{Methodology}

\subsection{Patients and Methods}

A retrospective study was performed on 106 patients who were subjected to VCUG scan at King Abdulaziz University radiology department. The range of the age of subject groups were varies from Newborn (up to 1 year) to Teenage (13 -15 years). VCUG scan was performed by utilizing PHILIPS and SEIMENS machines. DMSA radiopharmaceutical was administrated intravenously to the patient. Data records and information regarding patients have Voiding cystourethrography (VCUG) and ultrasound at the Department of General X-ray and Dimercaptosuccinic acid (DMSA) scintigraphy at the Department of nuclear medicine both were collected.

Table 2. Effective dose and imaging types.

\begin{tabular}{cc}
\hline Imaging & Effective dose (mSV) \\
\hline Voiding cycstourethrography & $0.14-1.56[19]$ \\
DMSA scan & $1.10-1.18[20]$ \\
\hline
\end{tabular}




\subsection{Study Area}

General X-ray department and nuclear medicine department at King Abdulaziz University Hospital (KAUH).

\subsection{Study Subjects}

The study group included patients who have a record of voiding cystourethrography (VCUG), ultrasound and dimercaptosuccinic acid (DMSA) scintigraphy.

\subsection{Study Design}

Retrospective study to analyze and compare the data records of voiding cystourethrography (VCUG) and ultrasound both done at the Department of General X-ray and Dimercaptosuccinic acid (DMSA) scintigraphy done at the department of nuclear medicine.

\subsection{Sample Size}

Up to 100 patients records will be analyzed in this study.

\subsection{Data Collection}

Electronically and hard copy data record of VCUG, US and renal scintigraphy results including patients details, protocols, indications, diagnosis, finding, ...etc. will be collected.

\section{Result}

\subsection{The Frequency Distribution of the Finding of Vesiocoureteral Reflux in Infants and Children}

In this study the VCUG scan is performed to find out the possibilities of renal scintigraphy and ultrasound in detecting vesicoureteral reflux (VUR) in infants and children. Out of the 106 patients, we found that the VCUG scan detect 65 cases with VUR in different grade levels while 41 cases were normal. Among the children with VUR, 39 had high-grade reflux (grade IV or V) while 26 had low-grade reflux (grades I, II or III). Figure 7 shows the finding of Vesiocoureteral reflux in infant and children.

\subsection{Frequency of Gender in Relation with VCUG Scans Study Findings}

$31 \%$ of female and $69 \%$ of male participated in this study. Figure 8 shows the distribution of gender in relation with VCUG scan study findings, we found that 20 female out of 39 had positive exam findings, while 19 had the negative findings and that represented $51 \%$ positive of the subject group and $49 \%$ were negative for female group.

For Male group, the scan findings were 44 positive out of 69 , and 25 were negative, which meant that $64 \%$ of the male subject group were having VUR, and 36\% were normal of the overall male subject group.

\subsection{Frequency of Age Groups and VCUG Scans Findings}

Figure 9 and Table 3 and Table 4 show that the age group from 1 - 3 years and from 3 - 9 for both gender

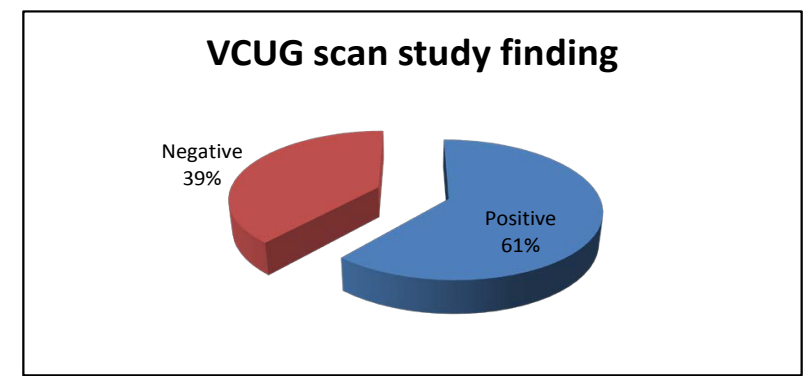

Figure 7. Vesiocoureteral reflux finding in infants and children. 


\section{Gender in relation with VCUG scan finding}

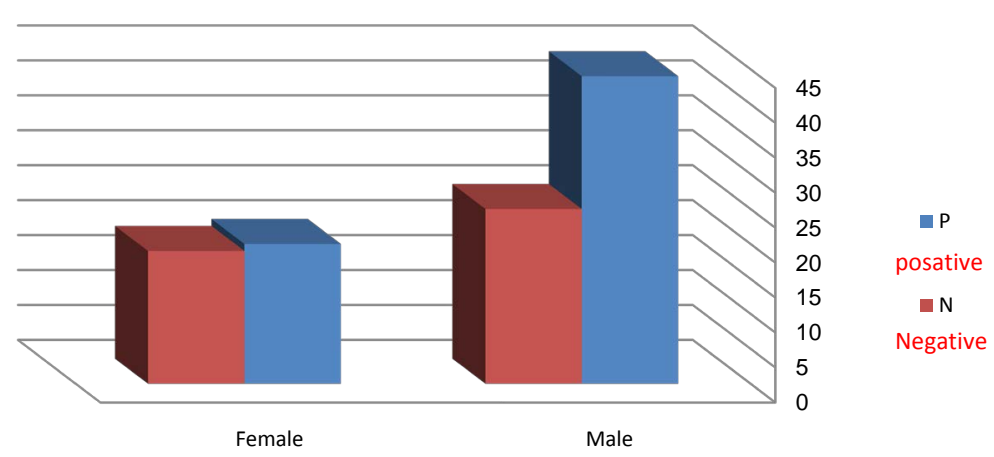

Figure 8. Distribution of gender in relation with VCUG scan study findings.



Figure 9. Frequency of age group distribution.

Table 3. Male cases.

\begin{tabular}{cccc}
\hline Age group & Total No. & Positive & Negative \\
\hline Newborn, 1 yr & $\mathbf{1 5}$ & 9 & 6 \\
Toddler, 1 yr -3 yr & $\mathbf{2 4}$ & 15 & 9 \\
Child, 3 - 9 yr & $\mathbf{2 0}$ & 13 & 0 \\
Preteen, 9 - 12 yr & $\mathbf{4}$ & 4 & 1 \\
Teenage $13-15$ yr & $\mathbf{4}$ & 3 & 7 \\
\hline
\end{tabular}

Table 4. Female cases.

\begin{tabular}{cccc}
\hline Age group & Total No. & Positive & Negative \\
\hline Newborn, 1 yr & $\mathbf{3}$ & 2 & 1 \\
Toddler, 1 yr -3 yr & 7 & 5 & 10 \\
Child, 3 - 9 yr & $\mathbf{2 1}$ & 1 & 3 \\
Preteen, 9 - 12 yr & $\mathbf{4}$ & 2 & 2 \\
\hline
\end{tabular}


represented the highest frequencies for positive VCUG scan study with $31 \%$ and $36 \%$ frequency, while Preteen and Teenage represented the lowest frequency for positive VCUG scan study with $8 \%$ frequency for each.

\subsection{Frequency of Clinical Reasons of Examination Distribution According to VCUG Scans Study Findings}

In regard of VUR incidence due to hydronephrosis \& Renal pelvis dilatation, recurrent UTI, history of VUR, neurogenic bladder, posterior urethra valve (PUV), minimal fullness of renal pelvis, and other pathology, the studies showed that hydronephrosis \& renal pelvis dilatation was the highest frequency reason for VCUG scan study and represent $36 \%$ of the overall cases with $53 \%$ positive finding, while minimal fullness of renal pelvis represented the lowest percent with $3 \%$ frequency and $0.0 \%$ positive finding. The frequency of recurrent UTI represent was $22.6 \%$ with $83 \%$ positive finding. Children with history of VUR represent $15 \%$ frequency and $81 \%$ positive finding, neurogenic bladder represent $9.3 \%$ frequency and $70 \%$ positive finding, posterior urethra valve (PUV) represent $7.5 \%$ frequency and $87.5 \%$ positive finding, other pathology represent $6.6 \%$ frequency and $57 \%$ positive finding. The frequency distribution shows in Figure $\mathbf{1 0 .}$

\subsection{Frequency and Correlation between VCUG Scan Finding and DMSA Scan Finding in Detecting the VUR}

Out of 106 patients participated in VCUG scan study, only 84 of them came back for a DMSA scan and that represented $69 \%$. We found 59 of the DMSA finding were same as VCUG finding and shows a renal scarring while 24 were normal study. Also, 53 patients participated in DTPA scan study and represented $50 \%$. We found 28 of the patients had obstructed kidney in the same side affected by the VUR and 25 were normal study.

\subsection{Frequency of the Urinary Tract Infection (UTI) and VUR}

Total of 52 patients participated in VCUG scan study during the period of UTI. We found that the urine culture result in 39 patients who diagnostic with VUR was positive and represented a high frequency with $75 \%$ while 13 was negative with frequency of $25 \%$. For DMSA scan study renal scarring is showing when patient has UTI. Out of 65 patients who had recurrent UTI, we found renal scarring in 48 of DMSA scan study and only 17 were normal study and represented with $74 \%$ and $26 \%$. Figure 11 shows the frequency of UTI and VUR finding in in both DMSA and VCUG studies.

\subsection{Frequency and Correlation between VCUG Scan Study Finding and Ultrasound Study Finding}

99 children out of 106 had ultrasound scan study of abdomen and pelvis for evaluation the kidneys and collecting system and that represented $93 \%$ of the overall cases. 58 of the Ultrasound findings gave the same result as

\section{Reasons of examination}

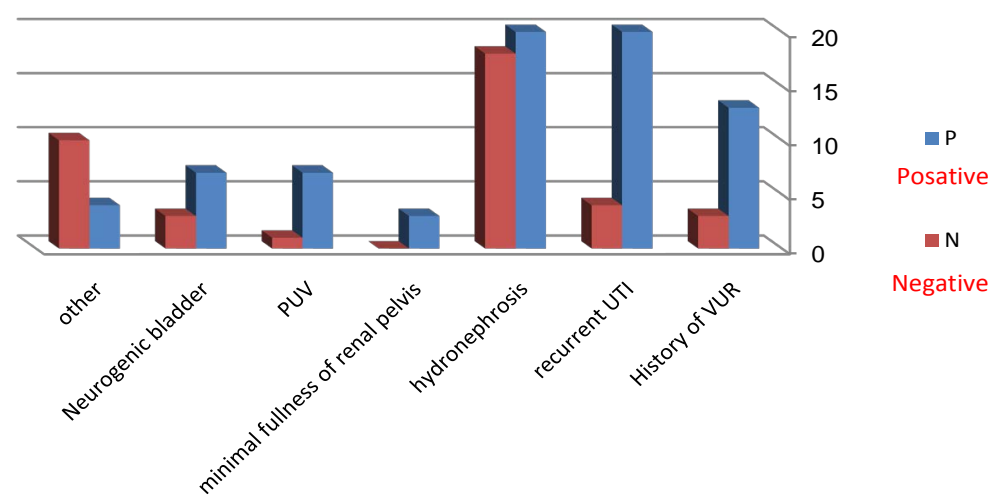

Figure 10. Frequency of clinical reasons of examination according to VCUG scans study findings. 
VCUG in detecting the VUR while 41 gave the opposite. Then we confirm it all with the Urine culture results of the same patients. The Urine culture result was 46 positive and 37 negative studies. Patients who has UTI (positive urine culture study) and positive ultrasound study were comparing with VCUG scan study, the Figure 12 shows that 25 of the children had abnormal US finding as the VCUG scan study during the period of UTI and represented $83 \%$ while only 5 had normal finding $17 \%$ of overall cases.

\section{Discussion}

In this study we will find out the possibilities of renal scintigraphy and ultrasound in detecting vesicoureteral reflux (VUR) in infants and children and if that can replace the voiding cystourethrography (VCUG). In regard with VUR incidence according to reason of examination, hydronephrosis \& Renal pelvis dilatation was the highest frequency reason for VUR scan study and represent $36 \%$ of the overall cases with $53 \%$ positive finding, while Recurrent UTI frequency was $22.6 \%$ with $83 \%$ positive finding, and patients who had history of VUR comes at the third place with 15\% frequency and 81\% positive finding. VUR was found in 65 of 106 children represented $61 \%$. Male children are more likely to diagnostic with VUR than female and they represented $69 \%$ while $31 \%$ for female. Among the children with VUR, 39 had high-grade reflux (grade IV or V), while 26 had low-grade reflux (grades I, II or III).

VCUG has many risks that may cause complications like secondary infection due to insertion of a vesical catheter and hypersensitivity of the contrast media. It's also an invasive unpleasant procedure that involves ionizing radiation exposure to the pelvis area. Instead of VCUG, renal scintigraphy and ultrasound scans can be used

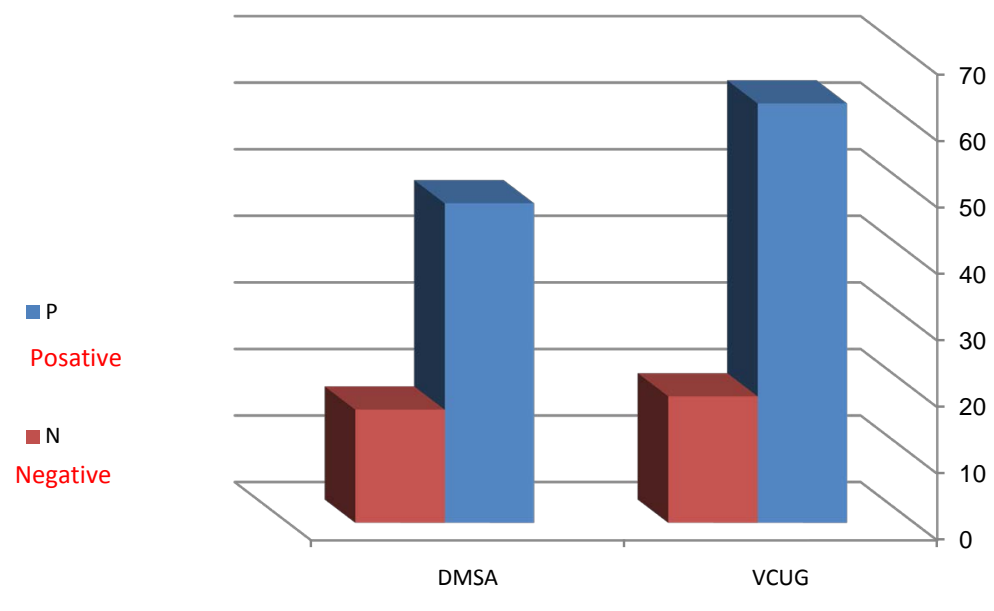

Figure 11. Frequency of the urinary tract infection (UTI) and VUR finding.

\section{US finding during UTI}

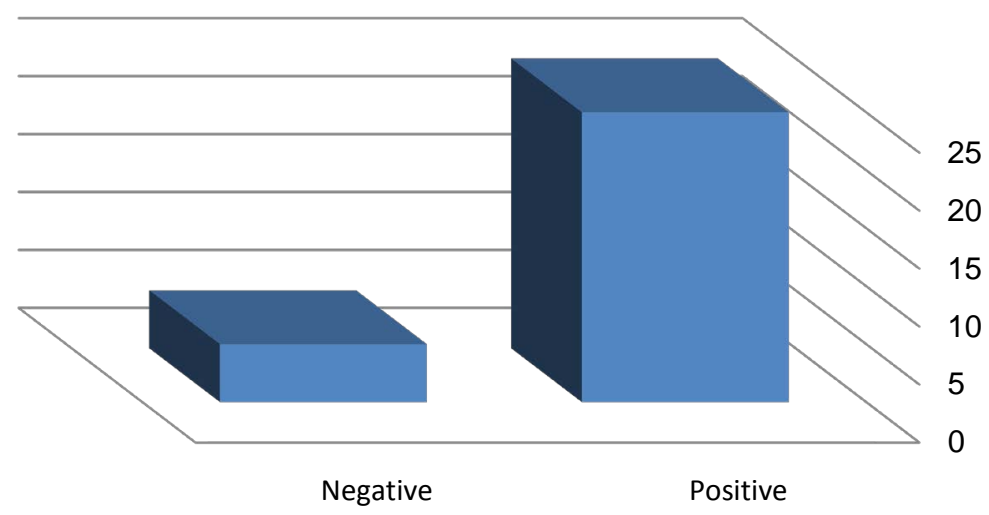

Figure 12. Urinary tract infection during US study. 
to predict VUR. When we classified VUR into low-grade and high-grade, we found the majority of children with high-grade VUR had an abnormal DMSA and represented 77.4\%, while 13 children with low-grade VUR had normal study. The DMSA scan study is more sensitively in detecting VUR with high-grade more than lowgrade. In recent study by Hyun Chong Ki "The incidence of VUR with a normal DMSA scan result was very low, and the degree of VUR in those children was low-grade and clinically insignificant." [27] and another study by Tseng et al. said that " 142 children with VUR and reported that all the children with grades III to V VUR (21 patients) had an abnormal DMSA scan result" [28]. These studies are agreed with the present study, most children with normal DMSA scan finding had low grade reflux. In this study, 48 of 65 children (74\%) the DMSA scintigraphy shows abnormal findings during the period of UTI while the VCUG scan study shows VUR in 75\% of the children during the period of UTI. The DMSA scan study results agree with Hyun Chong Ki, in his study "abnormal DMSA scintigraphy results were found in 72.5\% of children with febrile UTI" [27] but the high frequency of VCUG scan study doesn't agree with his study that have reported "VUR was found in $33.8 \%$ of the children with acute febrile UTI" [27].

Comparing results of ultrasound and VCUG scan, 59\% of the ultrasound findings gave the same result as VCUG in detecting the VUR in children. Then we confirm it all with the urine culture results of the same children. We found the majority of children with VUR had abnormal US during the period of UTI and represented $83 \%$.

\section{Conclusion}

VCUG is a sensitive, physiologic, and direct technique to assess hydronephrosis, Renal pelvis dilatation and Recurrent UTI in children especially male children from 1 - 9 years old. DMSA scan is useful in detecting high-grade reflux. In case of DMSA study showing normal finding (negative), VCUG is not recommended, because it rarely could be VUR. US performed during UTI may prove to be useful in detecting VUR.

\section{Recommendation}

Children specially with age groups from 1 - 9 years and who suffer of hydronephrosis, Renal pelvis dilatation, Recurrent UTI or have history of VUR are strongly recommended for VCUG scan to evaluation the VUR and grade of reflux. Children with normal DMSA scan finding during the period of UTI are not requiring a VCUG follow-up for the fact that they are rarely to have high-grade reflux. Further studies are needed in comparison with VCUG to find out the exact radiation dose that may harm the patient and to know the percentage of the clinically convenience of replacing VCUG exam.

\section{References}

[1] Bartholomew, M. (2012) Essentials of Anatomy and Physiology. Person.

[2] Marieb, E.N. and Hoehn, K. (2006) Human Anatomy and Physiology. 7th Edition, Person.

[3] Armstrong, P., Wastie, M. and Rockall, A. (2012) Diagnostic Imaging. 6th Edition, Wiley-Blackwell.

[4] Gross, G.W. and Lebowitz, R.L. (1981) Infection Does Not Cause Reflux. American Journal of Roentgenology, 137, 929-932. http://dx.doi.org/10.2214/ajr.137.5.929

[5] Smellie, J.M. and Normand, I.C.S. (1975) Bacteriuria, Reflux and Renal Scarring. Archives of Disease in Childhood, 50, 581-558. http://dx.doi.org/10.1136/adc.50.8.581

[6] Steinhardt, J.M., Kuhn, L.P. and Eisenberg, B. (1988) Ultrasound Screening of Healthy Infants for Urinary Tract Abnormalities. Pediatrics, 82, 609-612.

[7] Elder, J.S., Peters, C.A., Arant, B.S., et al. (1997) Pediatric Vesicoureteral Reflux Guidelines: Panel Summary Report on the Management of Primary Vesicoureteral Reflux in Children. Journal of Urology, 157, 1846-1851. http://dx.doi.org/10.1016/S0022-5347(01)64882-1

[8] Mattoo, T.K. (2011) Vesicoureteral Reflux and Reflux Nephropathy. Advances in Chronic Kidney Disease, 18, 348354. http://dx.doi.org/10.1053/j.ackd.2011.07.006

[9] Ansari, M.S., Gulia, A., Srivastava, A. and Kapoor, R. (2010) Risk Factors for Progression to End-Stage Renal Disease in Children with Posterior Urethral Valves. Journal of Pediatric Urology, 6, 261-264. http://dx.doi.org/10.1016/j.jpurol.2009.09.001

[10] Murawski, I.J. and Gupta, I.R. (2006) Vesicoureteric Reflux and Renal Malformations: A Developmental Problem. 
Clinical Genetics, 69, 105-117. http://dx.doi.org/10.1111/j.1399-0004.2005.00562.x

[11] Rubben, I., Goepel, M. and van Gool, J.D. (2011) Non-Neurogenic Bladder Dysfunction and Vesicoureteral Reflux in Children. Urologe A, 50, 551-556.

[12] Berrocal, T., López-Pereira, P., Arjonilla, A. and Gutié, J. (2002) Anomalies of the Distal Ureter, Bladder, and Urethra in Children: Embryologic, Radiologic, and Pathologic Features. Radiographics, 22, 1139-1164.

[13] Lebowitz, R.L., Olbing, H., Parkkulainen, K.V., Smellie, J.M. and Tamminen-Möbius, T.E. (1985) International System of Radiographic Grading of Vesicoureteric Reflux. Pediatric Radiology, 15, 105-109. http://dx.doi.org/10.1007/BF02388714

[14] Lim, R. (2009) Vesicoureteral Reflux and Urinary Tract Infection: Evolving Practices and Current Controversies in Pediatric Imaging. American Journal of Roentgenology, 192, 1197-1208. http://dx.doi.org/10.2214/AJR.08.2187

[15] http://www.radiologyinfo.org/en/photocat/gallery2.cfm?pid=1\&pg=voidcysto

[16] Howard, R.G., Roebuck, D.J., Yeung, P.A., Chan, K.W. and Metreweli, C. (2001) Vesicoureteric Reflux and Renal Scarring in Chinese Children. The British Journal of Radiology, 74, 331-334. http://dx.doi.org/10.1259/bjr.74.880.740331

[17] Lee, J.H., Kim, M.K. and Park, S.E. (2012) Is a Routine Voiding Cystourethrogram Necessary in Children after the First Febrile Urinary Tract Infection? Acta Paediatrica, 101, e105-e109. http://dx.doi.org/10.1111/j.1651-2227.2011.02507.x

[18] Easton, S. (2008) An Introduction to Radiography. Churchill Livingstone, London.

[19] Perisinakis, K., Raissaki, M., Damilakis, J., Stratakis, J., Neratzoulakis, J. and Gourtsoyiannis, N (2006) FluoroscopyControlled Voiding Cystourethrography in Infants and children: Are the Radiation Risks Trivial? European Radiology, 16, 846-851. http://dx.doi.org/10.1007/s00330-005-0072-6

[20] International Atomic Energy Agency (IAEA) (2006) Nuclear Medicine Resources Manual. IAEA, Vienna, 517.

[21] Riccabona, M. and Fotter, R. (2004) Urinary Tract Infection in Infants and Children: An Update with Special Regard to the Changing Role of Reflux. European Radiology, 14, L78-L88. http://dx.doi.org/10.1007/s00330-003-2030-5

[22] Elder, J.S., Peters, C.A., Arant Jr., B.S., et al. (1997) Pediatric Vesicoureteral Reflux Guidelines Panel Summary Report on the Management of Primary Vesicoureteral Reflux in Children. The Journal of Urology, 157, 1846-1851. http://dx.doi.org/10.1016/S0022-5347(01)64882-1

[23] Gearhart, J.G., Rink, R.C. and Mouriquan, P.D.E. (2009) Pediatric Urology. 2nd Edition, Elsevier Health Sciences, Amsterdam.

[24] Jequier, S. and Jequier, J.C. (1989) Reliability of Voiding Cystourethrography to Detect Reflux. American Journal of Roentgenology, 153, 807-810. http://dx.doi.org/10.2214/ajr.153.4.807

[25] Charsoula, A. (2014) Imaging of the Normal Male Urethra from the Neonate to the Elder: Radiologic Anatomy in Fluoroscopic Urethrography. ECR 2014/C-1524.

[26] Katzberg, R. (1992) Chapter 5. Urography, Cyctography and Urethrrography. In: Katzberg, R.W., Ed., The Contrast Media Manual, Lippincott Williams and Wilkins, Philadelphia, 62-63.

[27] Ki, H.C., Kim, S.O., Yoo, D.H. and Hwang, I.S. (2012) Abnormal Dimercaptosuccinic Acid Scan May Be Related to Persistence of Vesicoureteral Reflux in Children with Febrile Urinary Tract Infection. Korean Journal of Urology, 53, 716-720. http://dx.doi.org/10.4111/kju.2012.53.10.716

[28] Tseng, M.H., Lin, W.J., Lo, W.T., Wang, S.R., Chu, M.L. and Wang, C.C. (2007) Does a Normal DMSA Obviate the Performance of Voiding Cystourethrography in Evaluation of Young Children after Their First Urinary Tract Infection? The Journal of Pediatrics, 150, 96-99. http://dx.doi.org/10.1016/j.jpeds.2006.09.028 
Scientific Research Publishing (SCIRP) is one of the largest Open Access journal publishers. It is currently publishing more than 200 open access, online, peer-reviewed journals covering a wide range of academic disciplines. SCIRP serves the worldwide academic communities and contributes to the progress and application of science with its publication.

Other selected journals from SCIRP are listed as below. Submit your manuscript to us via either submit@scirp.org or Online Submission Portal.
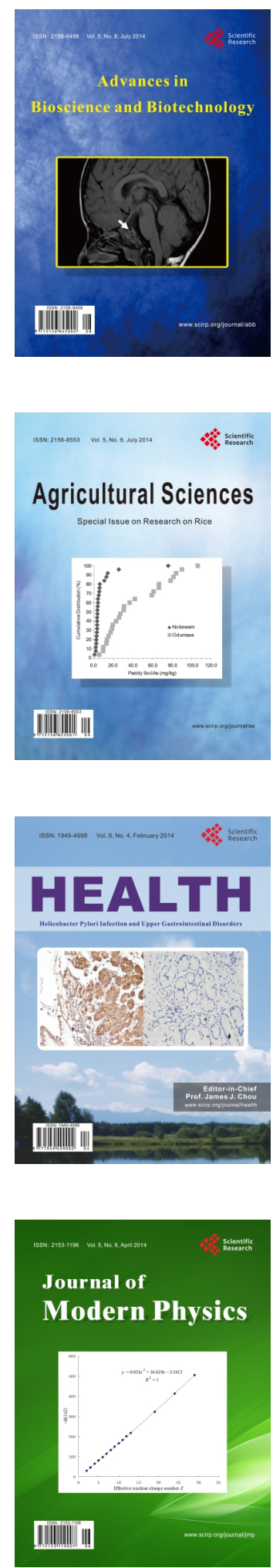
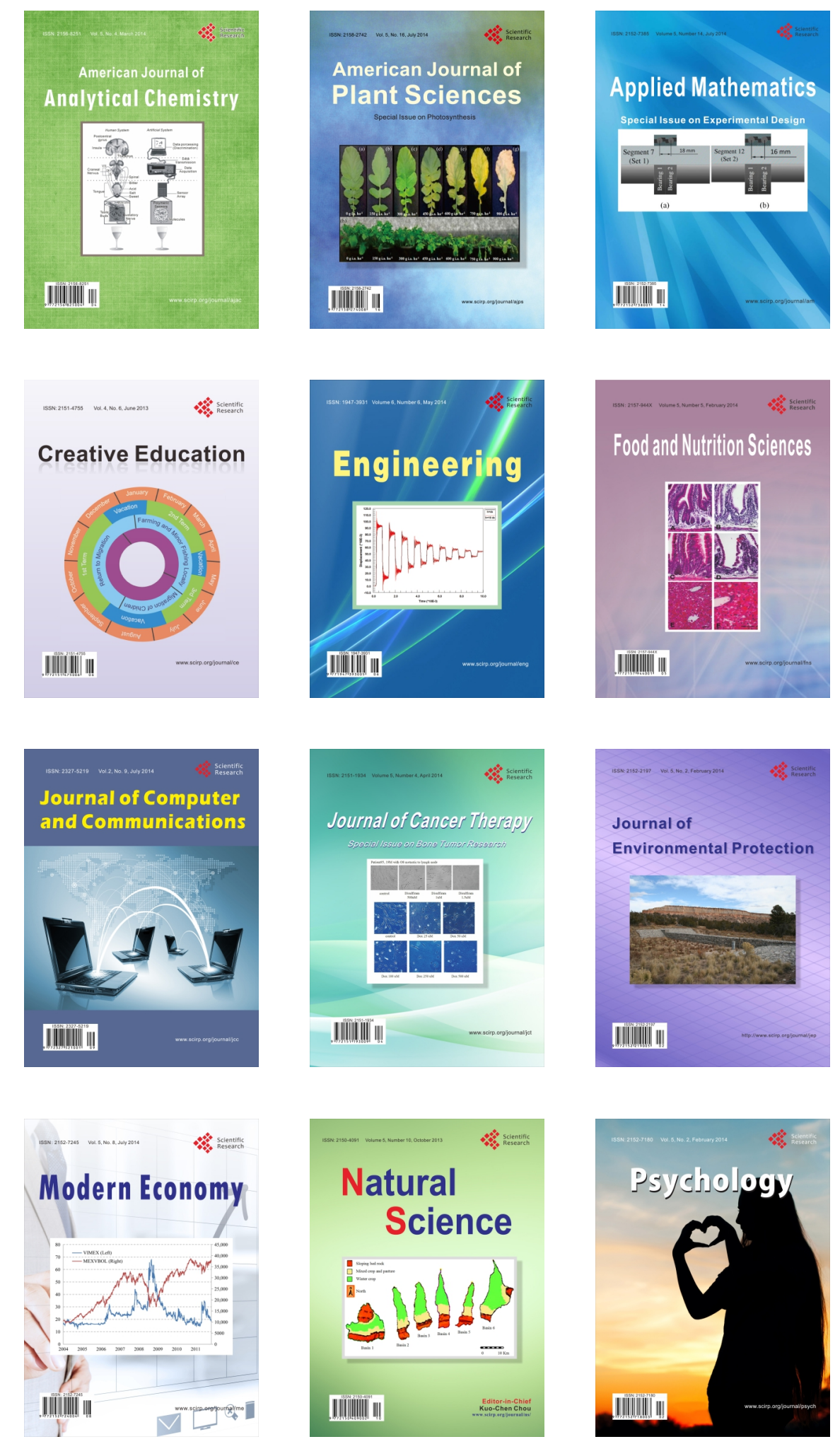